\title{
MANUEL DE PRATIQUES EN ÉDUCATION PHYSIQUE EN ÉDUCATION PROFESSIONNELLE ET TECHNOLOGIQUE UTILISANT L'APPLICATION GRATUITE EFE - EPTFIS
}

\section{ARTICLE ORIGINAL}

VIANA, Valderi Nascimento ${ }^{1}$, FECURY, Amanda Alves ${ }^{2}$, OLIVEIRA, Euzébio de ${ }^{3}$, DENDASCK, Carla Viana ${ }^{4}$, DIAS, Claudio Alberto Gellis de Mattos ${ }^{5}$

VIANA, Valderi Nascimento. Et al. Manuel de pratiques en éducation physique en éducation professionnelle et technologique utilisant l'application gratuite Efe Eptfis. Revista Científica Multidisciplinar Núcleo do Conhecimento. An 06, Ed. 04, Vol. 05, pp. 65-76. Avril 2021. ISSN: 2448-0959, Lien d'accès: https://www.nucleodoconhecimento.com.br/education-physics-fr/manuel-depratiques, DOI: 10.32749/nucleodoconhecimento.com.br/education-physics$\mathrm{fr} /$ manuel-de-pratiques

\section{RÉSUMÉ}

L'application, EFE-EduFisEPT, agit comme un soutien pour les activités de l'école d'éducation physique, en utilisant la pédagogie historique-critique se produit la confrontation de la connaissance scientifique de l'enseignant avec la connaissance empirique des élèves, provoquant le développement d'une nouvelle vision et l'action

\footnotetext{
${ }^{1}$ Éducateur physique, spécialiste en physiologie de l'exercice et nutrition sportive (FAMA), étudiant du programme d'études supérieures en éducation professionnelle et technologique (PROFEPT IFAP).

${ }^{2}$ Biomédical, $\mathrm{PhD}$ en maladies topiques, professeur et chercheur du cours de médecine du campus de Macapá, Université fédérale d'Amapá (UNIFAP).

${ }^{3}$ Biologiste, PhD en maladies tropiques, professeur et chercheur du cours d'éducation physique, Université fédérale du Pará (UFPA).

${ }^{4}$ Théologien, Ph.D. en psychanalyse clinique. II travaille depuis 15 ans avec la méthodologie scientifique (méthode de recherche) dans le cadre de l'orientation de production scientifique des étudiants à la maîtrise et au doctorat. Spécialiste de l'étude de marché et de la recherche axée sur la santé.

${ }^{5}$ Biologiste, Ph.D. en recherche théorique et comportementale, professeur et chercheur du cours de chimie de I'Institut d'éducation fondamentale, technique et technologique de l'Amapá (IFAP) et du Graduate Program in Professional and Technological Education (PROFEPT IFAP).
}

RC: 84007

Disponível em: https://www.nucleodoconhecimento.com.br/education-physics- 
dans la société. Le but de ce manuel est de guider le professeur d'éducation physique sur le contenu et les pratiques des cours d'éducation physique en EPT et sur la fonctionnalité de l'application gratuite EFE - EPTFis. Avec son interface simple, l'utilisation de l'application devient facile et avec des fonctionnalités intuitives. Ce produit éducatif existe comme un soutien pour l'enseignant à développer une éducation physique plus critique, qui comprend l'élève au-delà de l'être biologique, c'est-à-dire un être social, politique, culturel et historique. Dans lequel chaque classe, l'élève peut comprendre que le contenu présenté est lié à différentes dimensions de la réalité, contribuant à une vision de la société basée sur des valeurs et des attitudes envers l'objectif commun: la connaissance pour générer moins d'exclusions et de discriminations.

Mots-clés: Éducation physique, Pratique, EPT, Entraînement

\section{INTRODUCTION}

L'application, EFE-EduFisEPT, a été considérée comme un soutien aux activités scolaires d'éducation physique, visant à travailler dans son intégralité cette composante pédagogique, offrant ainsi une formation humaine, intégrale et omnilatérale aux lycéens de l'enseignement professionnel et technologique (EPT) (VIANA e DIAS, 2020).

Ainsi, en utilisant la pédagogie critique historique, les connaissances scientifiques de l'enseignant sont confrontées aux connaissances empiriques des élèves, ce qui entraîne le développement d'une nouvelle vision et d'une nouvelle action dans la société (SILVA, 2017; ANDRADE et al., 2020).

En plus du soutien pédagogique, l'application sert de moyen de diffusion des connaissances scientifiques, car toutes les classes sont basées sur cette forme de connaissance. Étant une application, il ya une plus grande facilité d'accès de n'importe quelle partie du monde (via les appareils de téléphonie mobile, tablettes,

RC: 84007

Disponível em: https://www.nucleodoconhecimento.com.br/education-physics- 
ordinateurs et autres), ce qui rend ce contenu accessible, rapprocher l'utilisateur de la communauté scientifique, mettre à jour leurs connaissances. II encourage l'utilisateur à être un connaisseur et ont un plus grand intérêt à effectuer un travail pédagogique différencié (SANTOS et al., 2018).

\section{OBJECTIF}

Guidez le professeur d'éducation physique sur le contenu et les pratiques des cours d'éducation physique en EPT et sur les fonctionnalités de l'application gratuite EFE EPTFis

\section{MATÉRIAUX ET MÉTHODES}

L'application décrite ici peut être téléchargée gratuitement https://play.google.com/store/apps/details?id=com.ifap.efe (Play Store), exclusivement pour Android. Le contenu complet peut être consulté gratuitement à la https://www.edufisept.com.br/ .

\section{L'APPLICATION ET SON CONTENU}

\section{L'APPLICATION}

L'application a été conçue, ainsi que le site Web, pour donner au professionnel qui travaille (ou non) avec l'éducation professionnelle et technologique (EPT) un outil théorique pratique afin de contribuer à la formation humaine, intégrale et omnilatérale des étudiants (VIANA et al., 2020).

La étape par étape qui suit vise à faciliter le travail des professionnels qui ont moins d'affinité avec ce type de technologie.

RC: 84007

Disponível em: https://www.nucleodoconhecimento.com.br/education-physics- 
La figure 1 affiche l'interface d'entrée de l'application. Pour afficher le menu, vous devez faire glisser l'écran de gauche à droite.

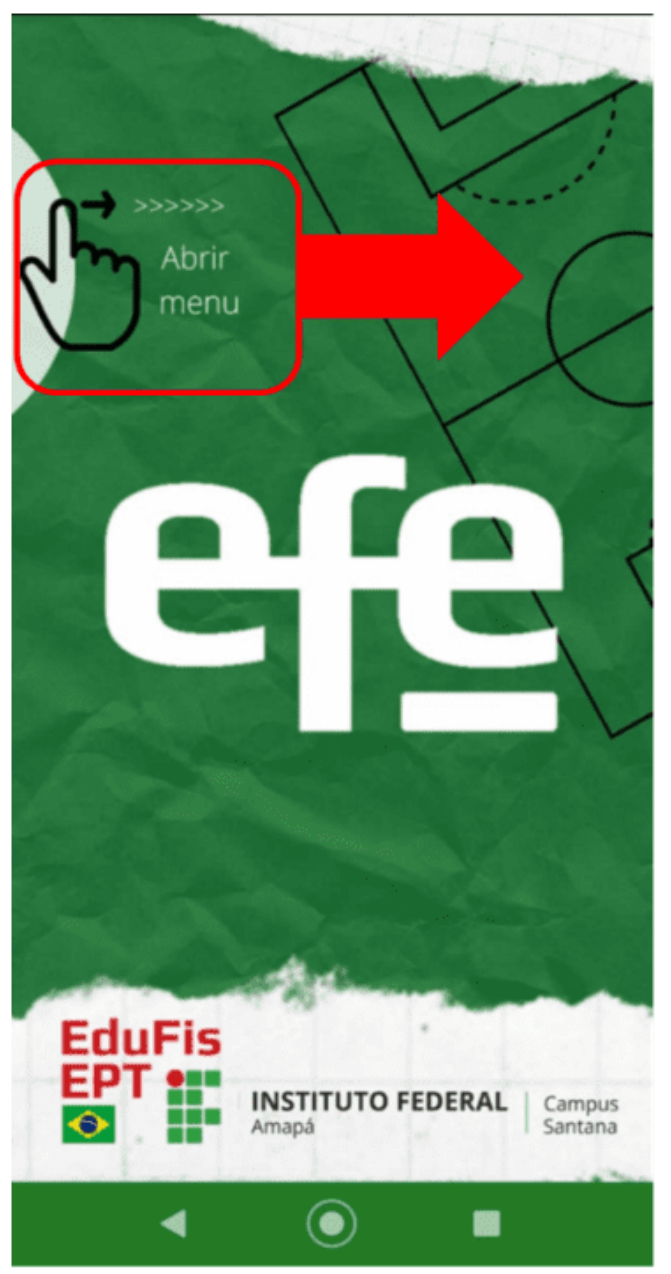

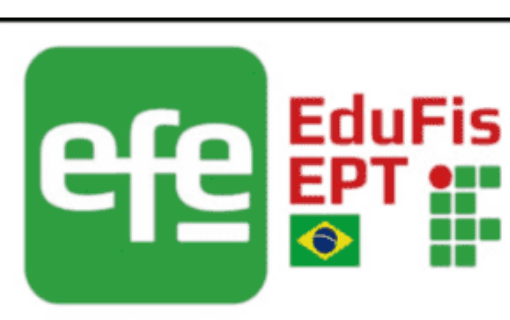

Práticas pedagógicas

Fechar tela

탕ㄱ Feedback

Quem somos

के Colabore

(4) Relatar um problema

I. Sair

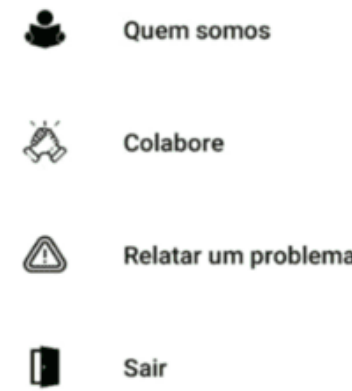

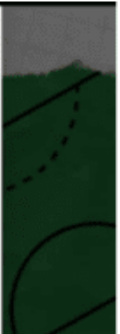

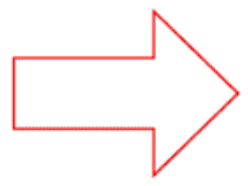

4

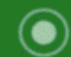

RC: 84007

Disponível em: https://www.nucleodoconhecimento.com.br/education-physics- 
La figure 2 montre ce qui se passe lorsque nous cliquons sur " Pratiques pédagogiques » dans le menu. Un autre écran s'ouvre où vous pouvez sélectionner le type de pratique.
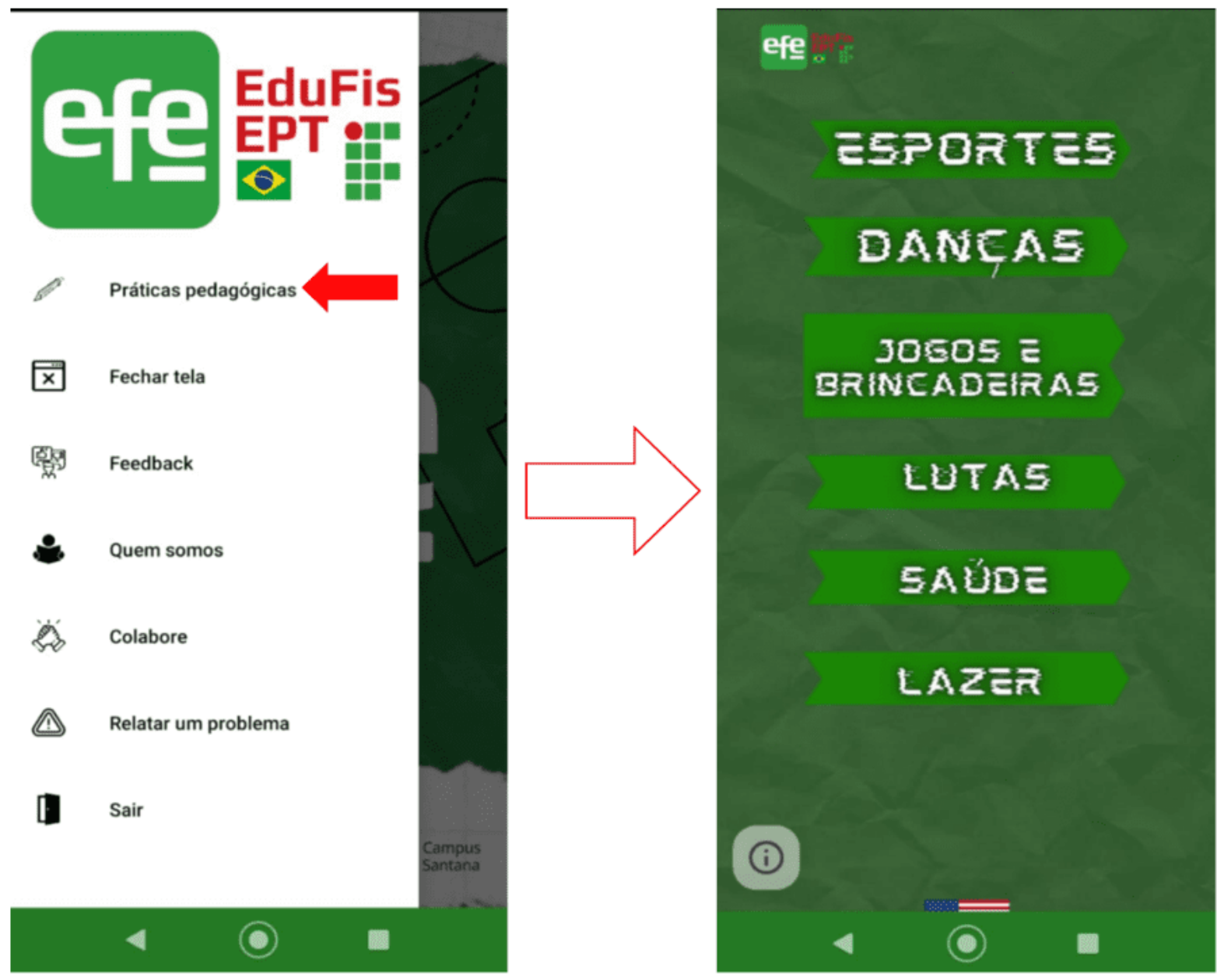

RC: 84007

Disponível em: https://www.nucleodoconhecimento.com.br/education-physics- 
La figure 3 montre qu'en cliquant sur chaque élément, il y a des boutons pour accéder à la théorie qui la compose et à la pratique elle-même, à mener avec les élèves.

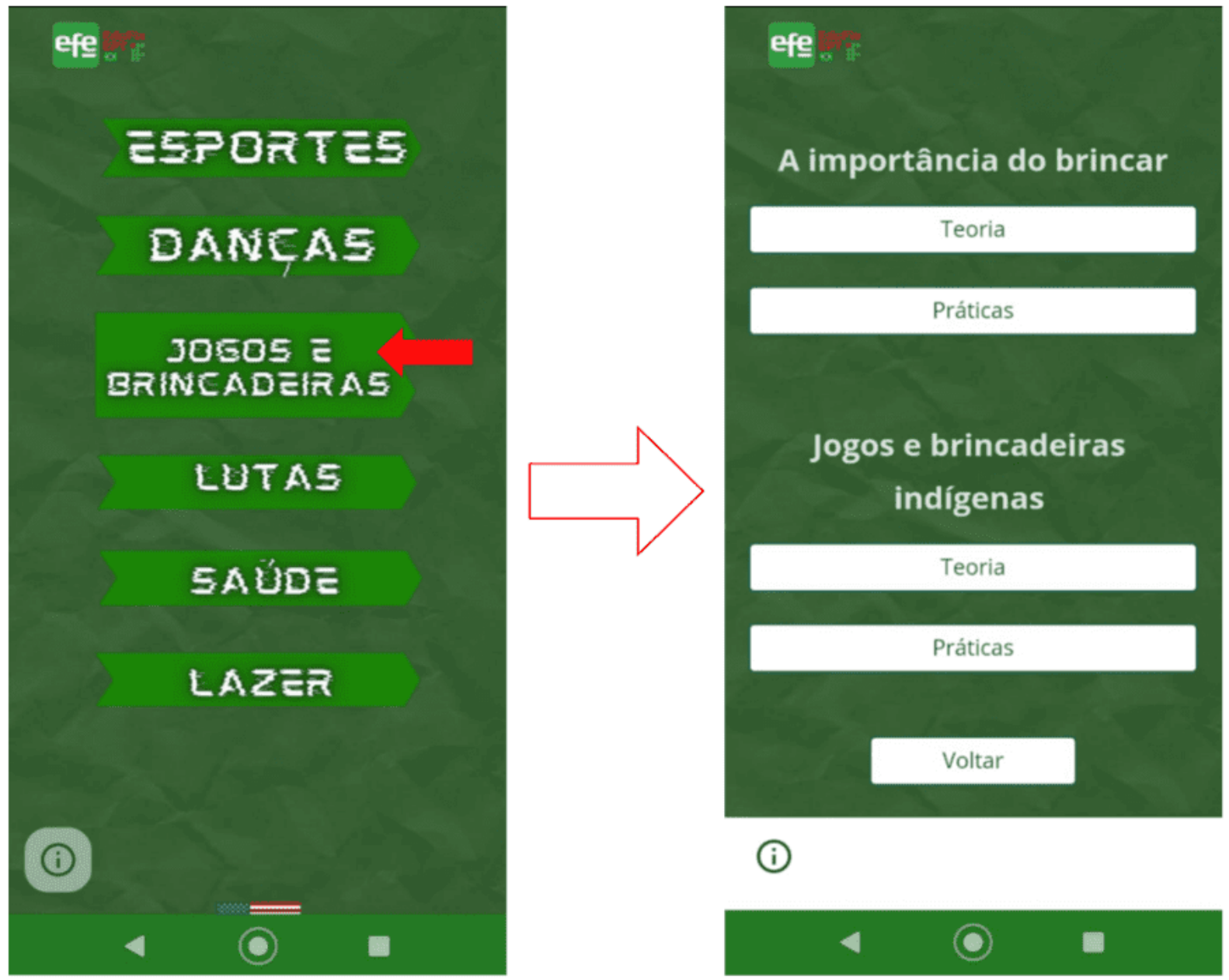

RC: 84007

Disponível em: https://www.nucleodoconhecimento.com.br/education-physics- 
La figure 4 montre qu'en cliquant sur "Théorie ", un onglet est mis à disposition avec la théorie raisonnée et aussi des documentaires vidéo pour une meilleure compréhension de la partie pratique.

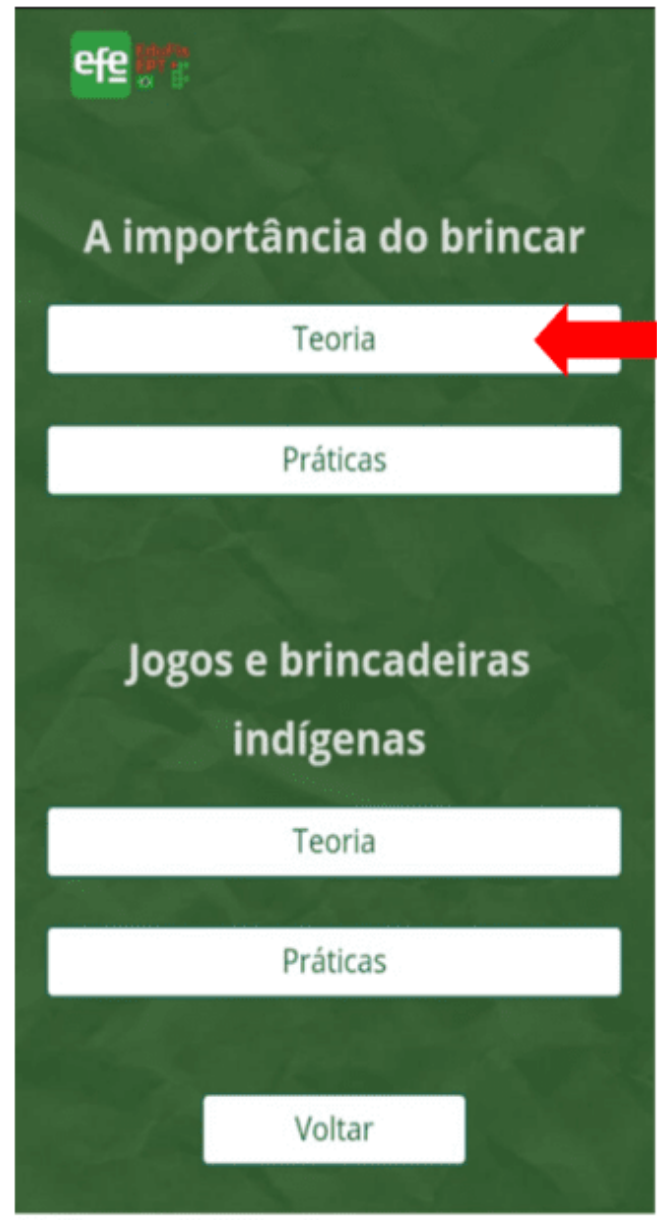

(i)

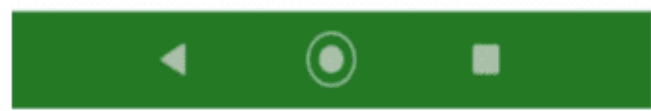

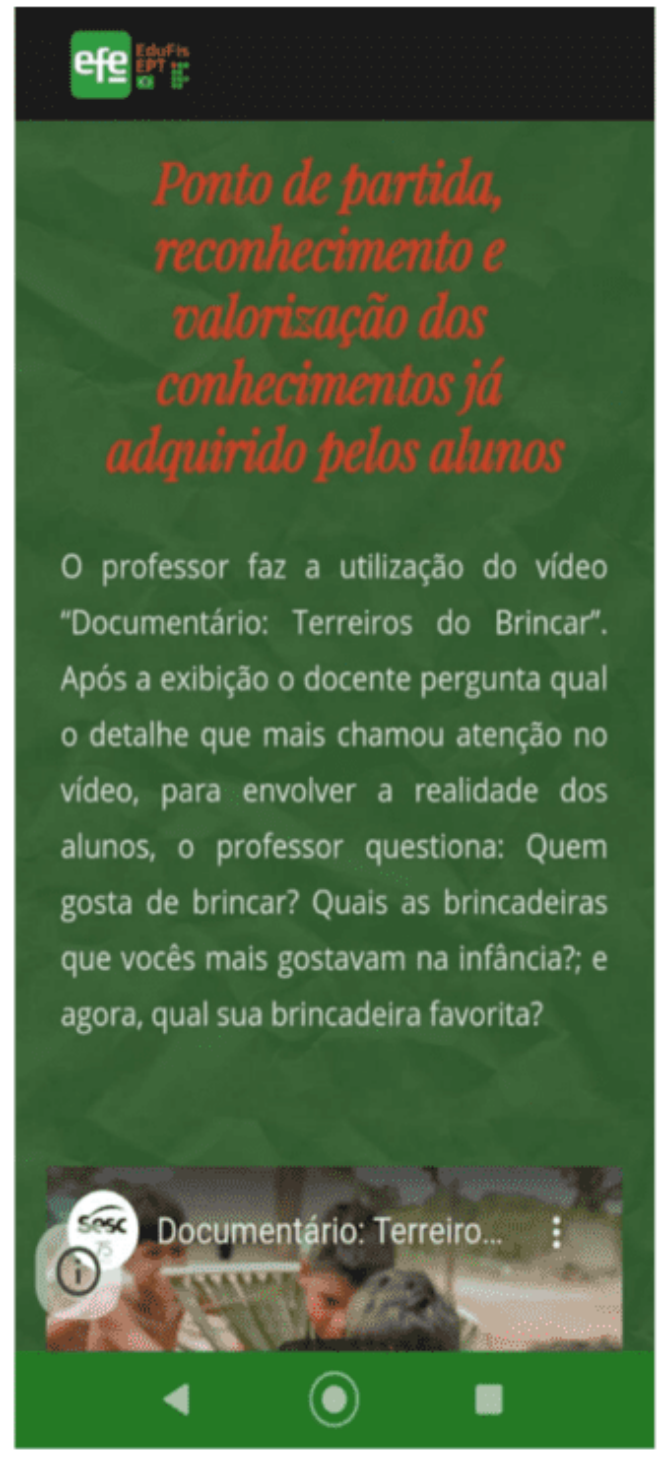

RC: 84007

Disponível em: https://www.nucleodoconhecimento.com.br/education-physics-

fr/manuel-de-pratiques 
La figure 5 montre qu'en cliquant sur «Pratique », un nouvel écran s'ouvre lorsque plusieurs pratiques possibles sont présentées.

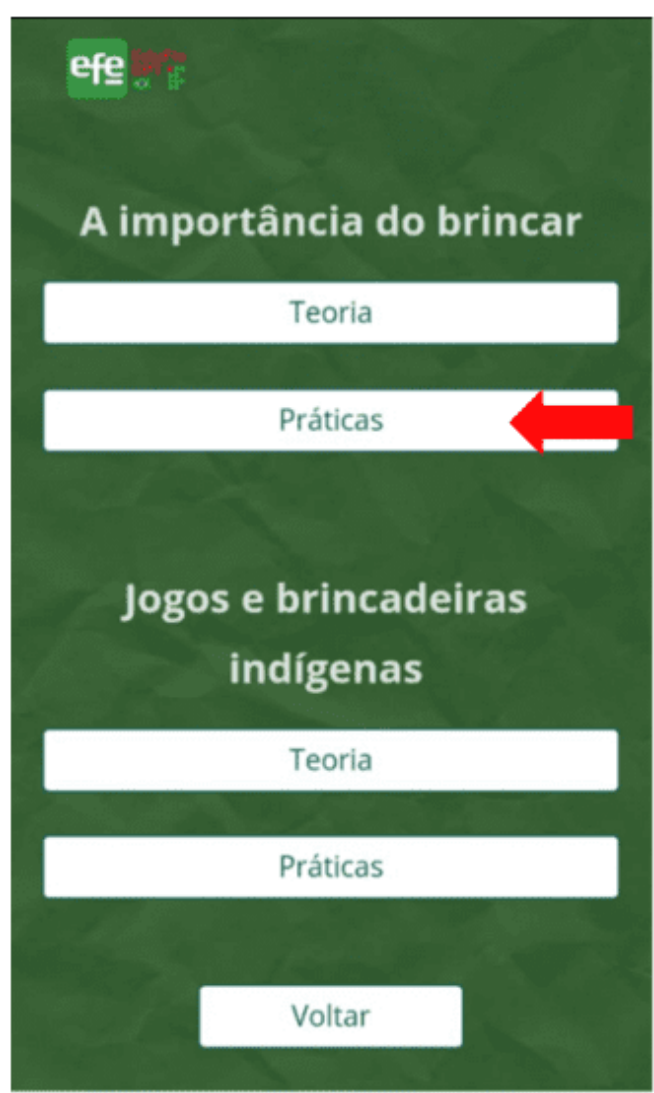

(i)

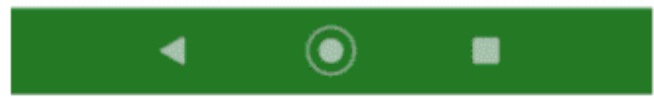

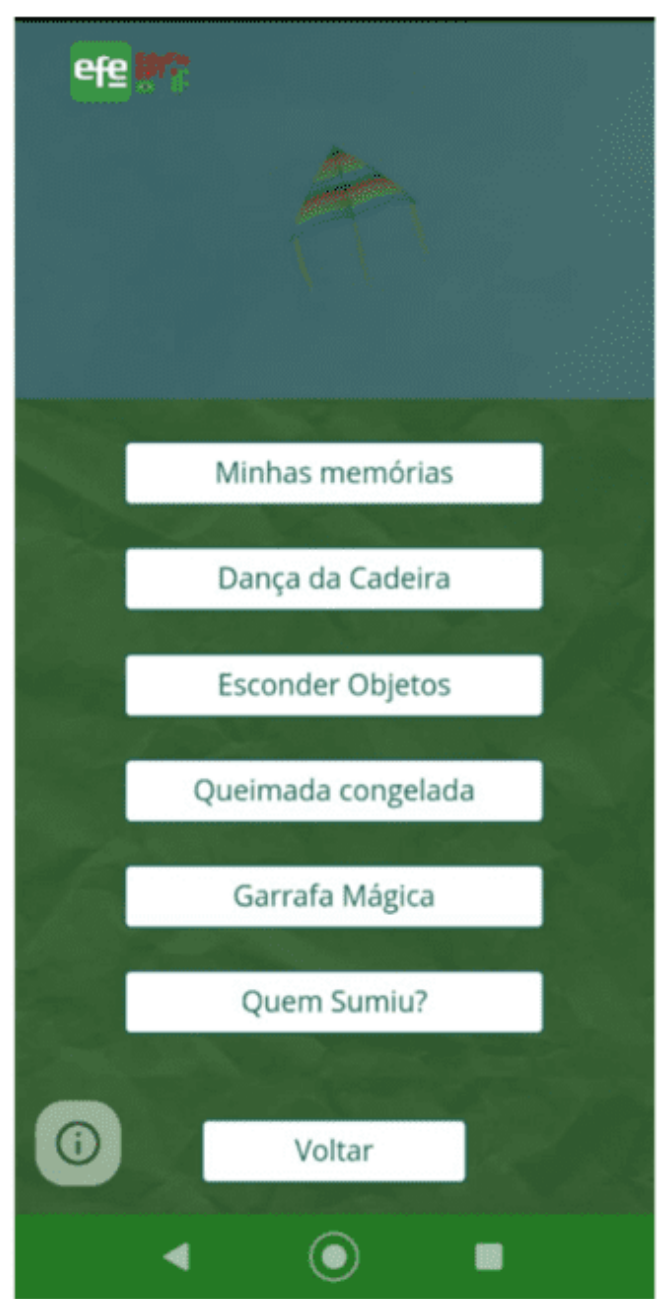

RC: 84007

Disponível em: https://www.nucleodoconhecimento.com.br/education-physics-

fr/manuel-de-pratiques 
La figure 6 montre qu'en cliquant, par exemple, sur " Queimada Congelada »( balle aux prisonniers ), un nouvel écran s'ouvre là où, pour chaque pratique, il y a un élément qui montre les ressources nécessaires pour cela, la description détaillée et un pourboire utile. Ce format se répète dans toutes les autres pratiques d'application.
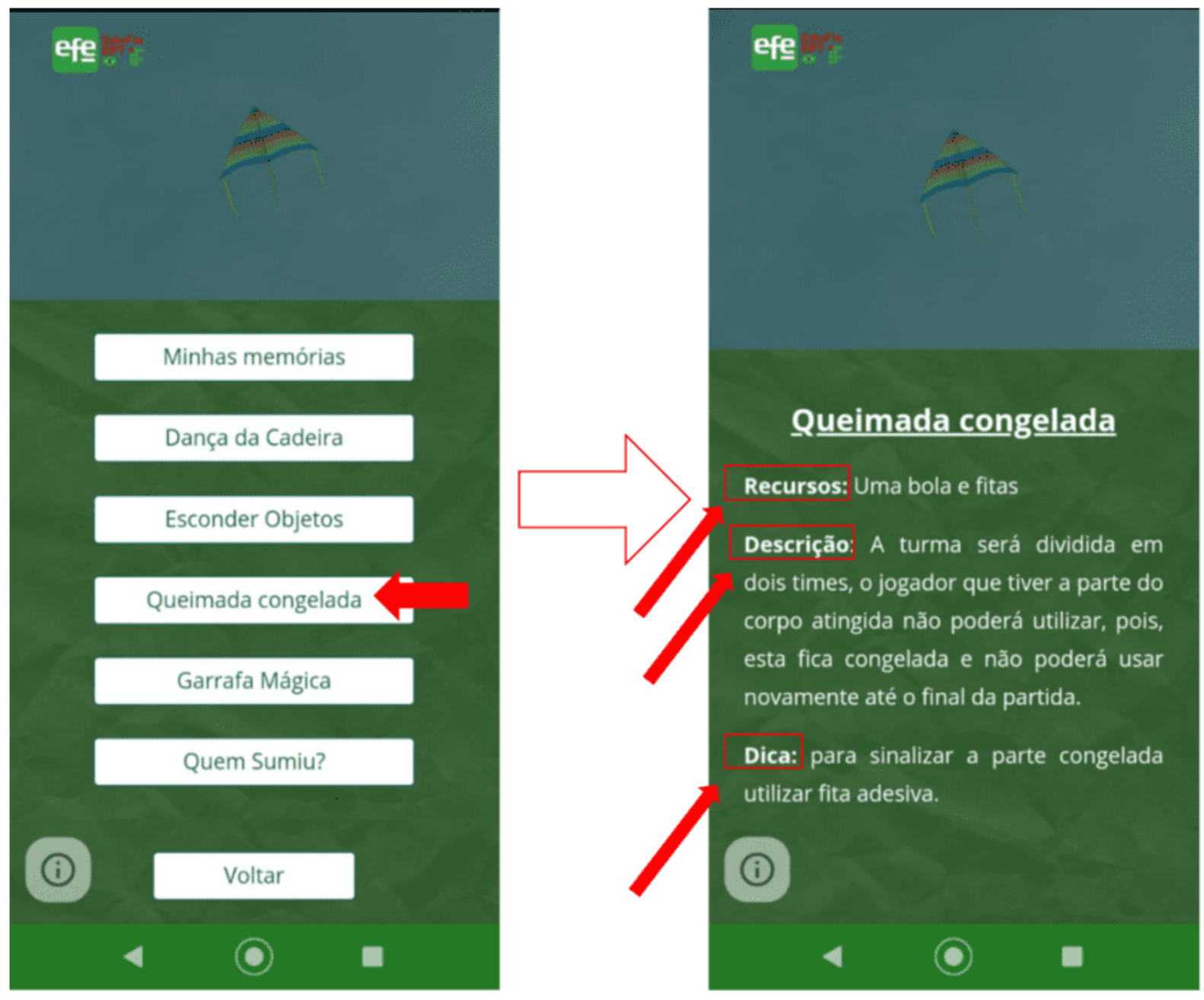

RC: 84007

Disponível em: https://www.nucleodoconhecimento.com.br/education-physics- 
Figure 7 Retour au menu de la maison, en cliquant sur " Feedback », ouvre un écran où l'utilisateur peut participer à une collecte de données, important pour la poursuite de la recherche de l'auteur de l'application, dans ce domaine de connaissances.

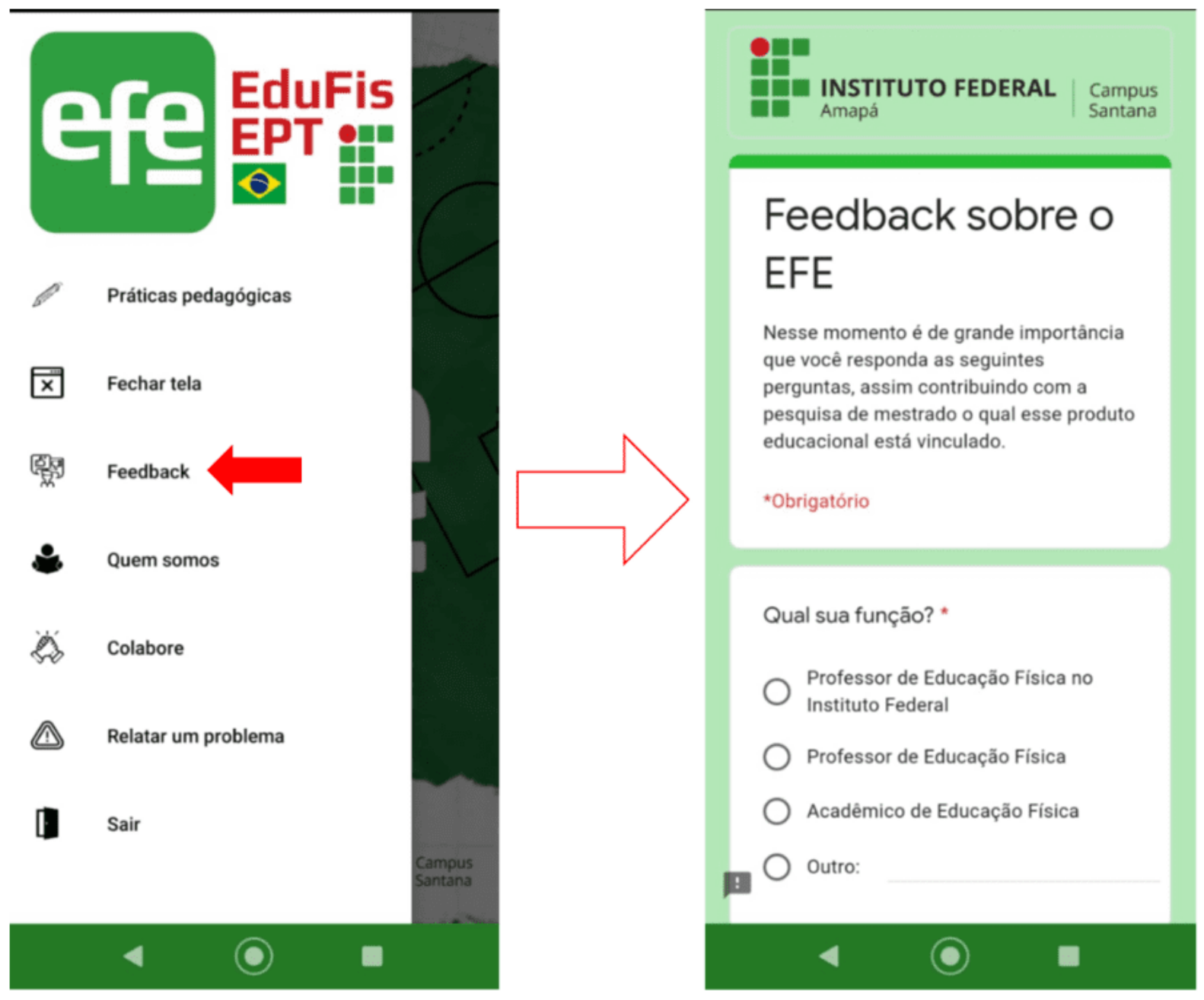

RC: 84007

Disponível em: https://www.nucleodoconhecimento.com.br/education-physics- 
Figure 8 En appuyant sur le bouton "Qui nous sommes ", toujours dans le menu principal, l'utilisateur aura accès aux informations des auteurs de l'application, ainsi qu'à leurs CV sur la plateforme Lattes.
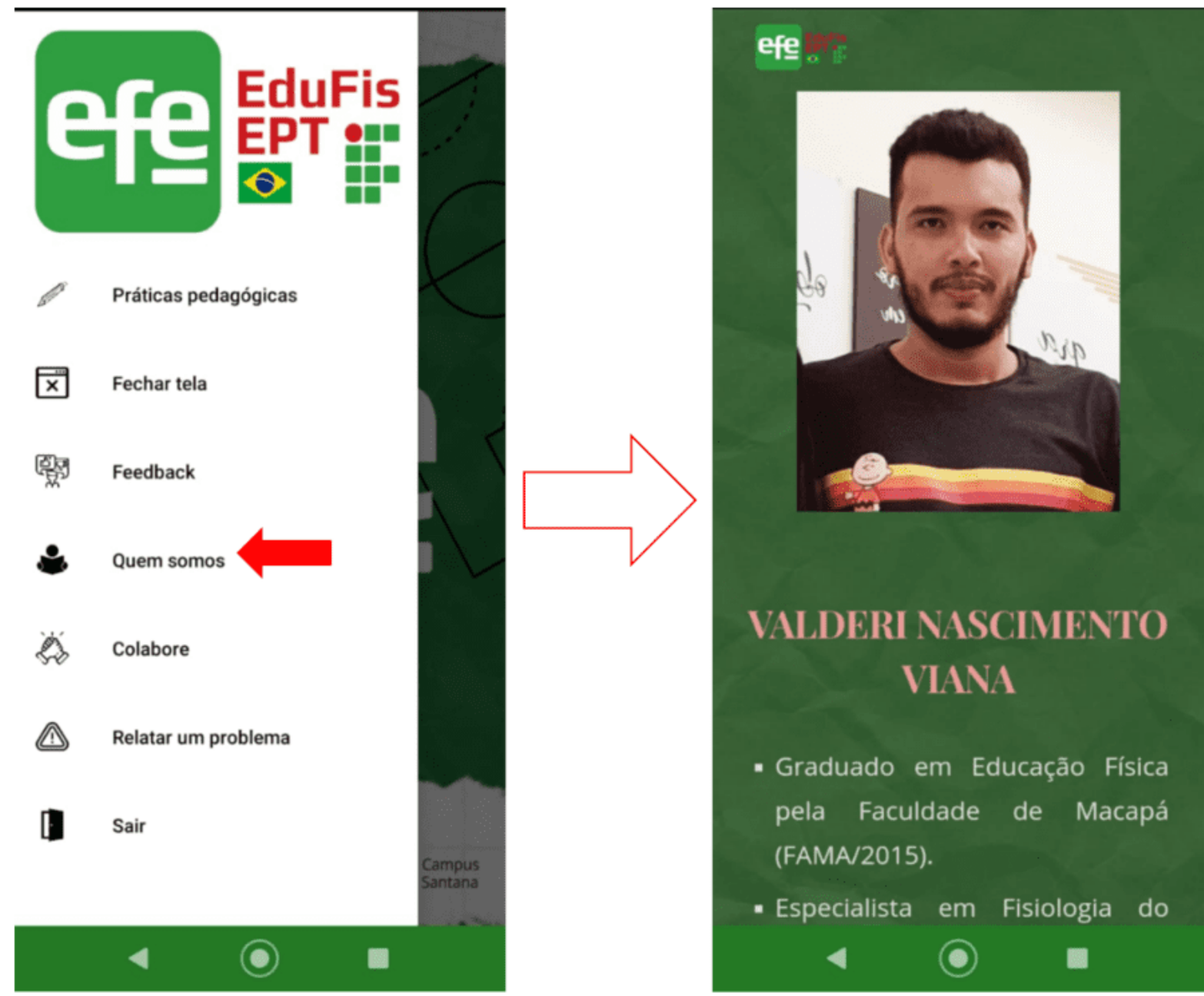

RC: 84007

Disponível em: https://www.nucleodoconhecimento.com.br/education-physics- 
Figure 9 Dans le menu, en cliquant sur "Collaborer », l'utilisateur peut collaborer à l'amélioration des connaissances au sein de cette APP et sur le site Web, l'envoi en format PDF ou DOC, avec sa propre pratique pédagogique et avec des évaluations dirigées par l'application. On tient compte du fait que la connaissance n'est pas construite de façon solitaire.
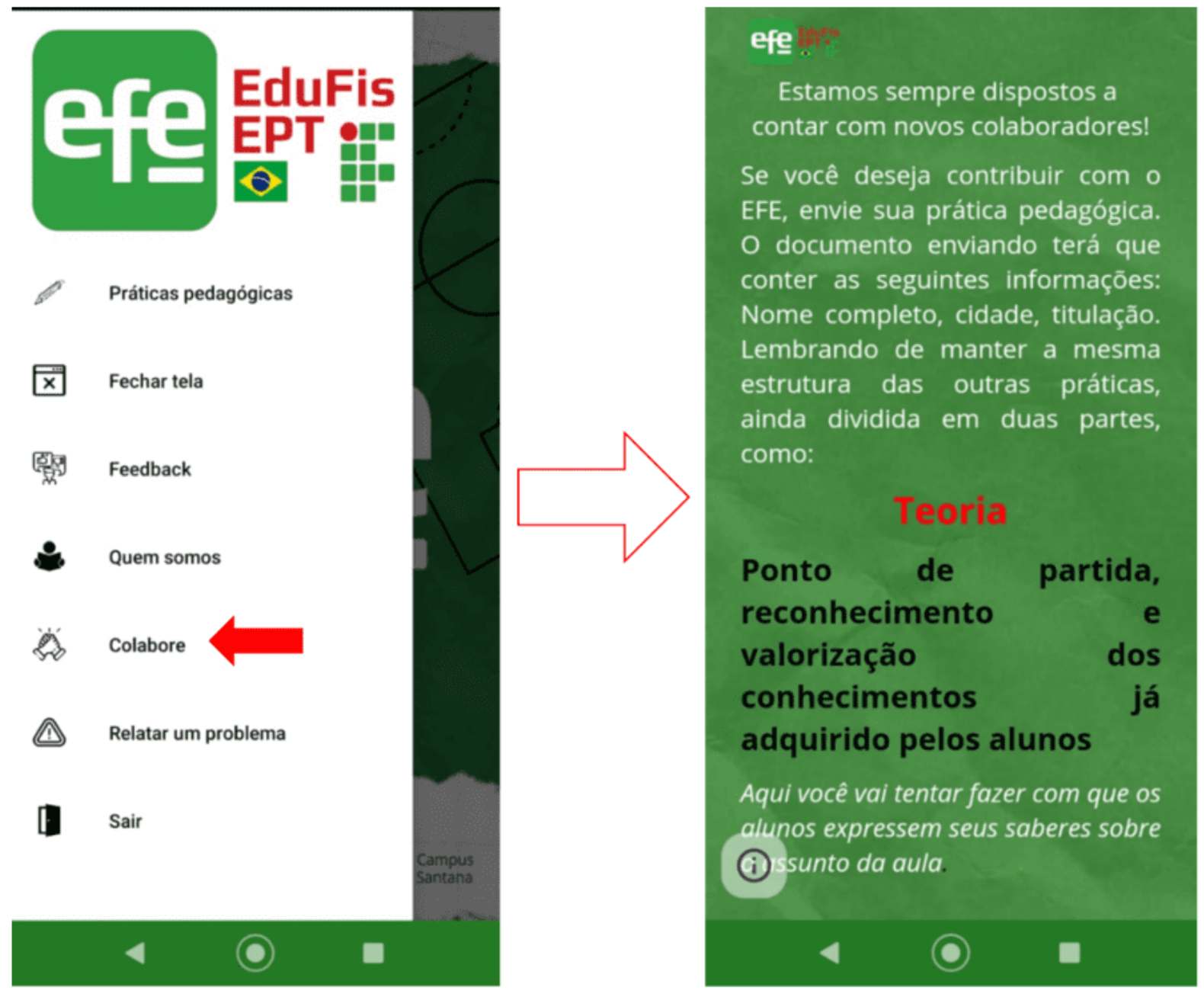

RC: 84007

Disponível em: https://www.nucleodoconhecimento.com.br/education-physics- 
Figure 10 Dans le menu, vous pouvez toujours cliquer sur " Signaler un problème » dans l'application, cliquer sur « Fermer la fenêtre » ou même cliquer sur « Exit ».

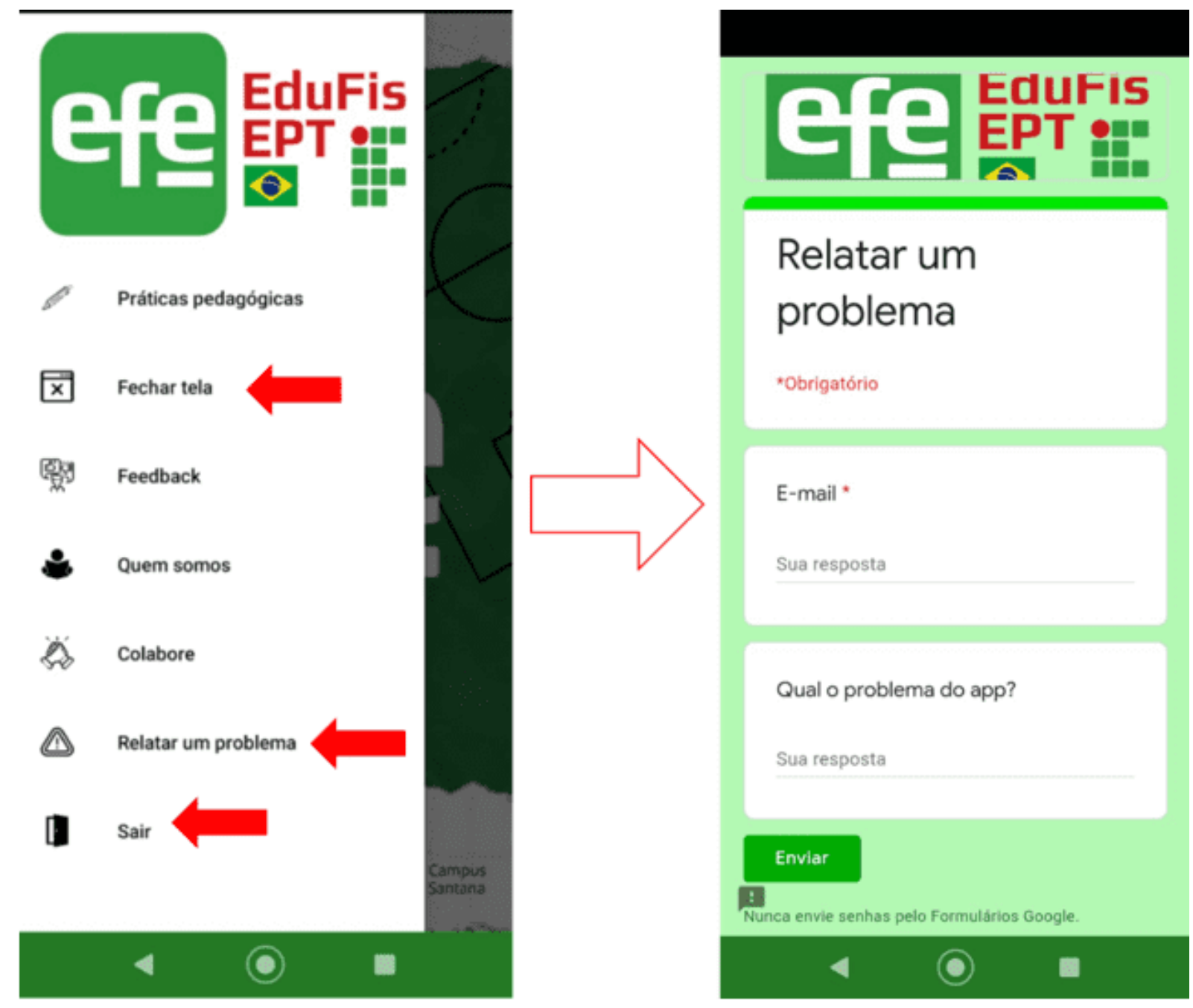

\section{LE CONTENU}

Avec un accès facile et rapide via l'application Android ou via le site, le contenu a l'ambition de montrer la partie théorique de la formation humaine, intégrale et omnilatérale de toutes les activités pratiques disponibles.

RC: 84007

Disponível em: https://www.nucleodoconhecimento.com.br/education-physics- 
Pour cela, il avait un cadre théorique étudié par l'auteur au cours de son programme d'études supérieures stricto sensu, ainsi que la pratique également dérivée de la recherche, beaucoup effectuée au niveau régional.

Figure 11 Montre une partie de la théorie dans l'article « Jeux et jeux ».

\section{Jogos-e-brincadeiras?l}

Teoriaq

Ponto de partida, reconhecimento e valorização dos conhecimentos · já adquirido pelos alunos

ฯ

O professor faz a utilização do vídeo "Documentário:- Terreiros do- Brincar". (https://www.youtube.com/watch?v=29yJW7XQUws- -). Após· a- exibição- o- docentepergunta qual o detalhe que mais chamou atenção no video, para envolver a realidade dos. alunos, o professor-questiona:-Quem gosta-de brincar?-Quais as brincadeiras que vocêsmais gostavam na infância?; e-agora, qual sua brincadeira favorita?

\section{Como pratica-social, questões que podem'ser resolvidas/debatidas} com o auxilio deste conteúdo. sugestões das dimensões teórico-práticas:-ศा

-Conceitual: O que-são-brincadeiras? -

- Cultural: $\mathrm{O}$ brincar-é-só coisa de criança? $\uparrow$

RC: 84007

Disponível em: https://www.nucleodoconhecimento.com.br/education-physics- 
-Psicológica/Histórica:-Qual-a-importância-do-brincar?ף

-Social:Por-que na-vida-adulta-somos-obrigados-a esquecer-as-brincadeiras?-

ๆ

Encaminhamento-metodológico para-a-explicação-do-conteúdo

Exibição-integral-ou parcial- do- documentário- "Tarja-Branca". - Disponivel-em. https://www.videocamp.com/pt/campaigns/quarentena-believe-

tarjabranca/player?special_id $=84100 \ldots$

O professor-vai fazer uma reflexão sobre o documentario e abordar assuntos como: brincadeira e a vida adulta; como - mercado-de trabalho-subtrai-das pessoas os momentoslúdicos-e-a-importância-dessa-pratica-para-a-saúde, fazendo-sempre-uma-ligação-com-ovideo exibido -1

\section{Formação,-atitudes-e-valores}

Ao final, o aluno tenha a atitude de perceber que mesmo deixando a infäncia nunca. parou-de-brincar, apenas -mudou- 0 -modo-e formas. Durante essa pratica-trabalha-com-0. aluno-a-cooperação, uniâo, senso-critico-e-criatividade.

$\mathrm{RC}: 84007$

Disponível em: https://www.nucleodoconhecimento.com.br/education-physics- 
La figure 12 montre certaines des pratiques étudiées et décrites dans l'article « Jeux et jeux ", toujours subdivisées en "Ressources 》 (nécessaires pour assembler la pratique), «Description » (méthode ou règle à suivre pour que la pratique fonctionne) et «Conseil » (toujours un autre point de vue sur la pratique).

\section{Minhas memórias \\ I \\ Recursos:-folha-de-A4, canetas}

Descrição: Em sala, -solicitar dos alunos que em uma folha-de-A4 faça um resgate de -suasmemorias as mais antigas, clara e-alegre, momentos de brincadeiras-de-sua infäncia. Podeser um brinquedo, um momento-especifico-como: uma-viagem, uma festa, férias. E-comisso, construir-a partir-dessas-lembranças como elas -se-conectam com-sua-vida-atual. -

Dicas:- Caso-0 aluno tenha-algum - registro,-no-grupo- de-WhatsApp-os alunos podemmandar a foto junto com um audio (Possivel ensino remoto), para-isso, o professor precisamarcar-um-dia-especifico.- Ou-pode-ser-feito-em-sala-de-aula, - o-qual-cada-um-leva-seuregistro-e-comenta- 9

\section{Danca'da.Cadeira,}

Recursos:-Cadeiras, caixa-de-som -

Descrição:- Será - formado - uma-roda-com-as - cadeiras-e - os alunos ficarão-a- sua-volta, contendo-uma-cadeira-a-menos que-o-número-de participantes. Ao-som-da-música-osalunos-vão-andar-ao-redor-da-roda-(dançando-no-ritmo-da-música) - Quando-a-músicapausar-cada-integrante-ocupa-uma-cadeira, - sempre-sobrando um-sem, ficando-fora-dadança.-A cada nova rodada fica sempre com uma cadeira a menos até sobrar apenas-umae-dois-alunos, que tentaram ocupá-1a, tornando-se-assim, - o vencedor. -

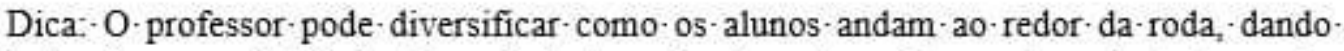
instruções como: Mais rápido; mais lento; andar-de costas; mão na cabeça; indo de acordo. com-a-criatividade-do professor. .

RC: 84007

Disponível em: https://www.nucleodoconhecimento.com.br/education-physics- 


\section{Quem·Sumiu?·}

Recursos:-Tecido, sala-de-aulaף

Descrição:-Em-sala-de-aula-ou-na-quadra-um-aluno-será-escolhido, antes-de-ter-os-olhosvendados, -vai-olhar para-a turma, - logo-depois-ficar-de-costas e-será-vendado, enquantoisso um aluno-selecionado-sai-do-local, 0 -aluno-com 05 -olhos cobertos-vira-de frente paraturma e tira-a-venda, com isso, terá que adivinhar quem sumiu do local - O que estava foraretorna e ocupa-o-lugar de quem deveria descobrir - 0 -integrante que estava-faltando. -

Dica: $O$-professor-pode autorizar-até-3 - características-do-aluno que-sumiu, tal-duvidasendo respondida pelos-alunos-da-turma-f

\section{Esconder-Objetos,}

Recursos:-20-Bolinhas-de plástico, -1

Descrição:A-turma-será-dividida-em-2-times. Uma-equipe-sai-da-sala-e pela-quadra-oupor-determinado espaço-dentro da-escola-tem que-esconder-os-objetos-(dentro-de-uns-3. minutos). Otime que ficou na-sala aguardando quando autorizado pelo professor -sai paraprocurar-os-objetos, por-um-tempo-limitado-Cada-objeto-encontrado-é-marcado-umapontuação para-o-time, essa pontuação pode-ser-definida pelas-cores-das-bolinhas. -

\section{CONCLUSIONS}

Avec son interface simple, l'utilisation de l'application devient facile et avec des fonctionnalités intuitives. Ce produit éducatif existe comme un soutien pour l'enseignant à développer une éducation physique plus critique, qui comprend l'élève au-delà de l'être biologique, c'est-à-dire un être social, politique, culturel et historique. Dans lequel chaque classe, l'élève peut comprendre que le contenu présenté est lié à différentes dimensions de la réalité, contribuant à une vision de la société basée sur des valeurs et des attitudes envers l'objectif commun: la connaissance pour générer moins d'exclusions et de discriminations.

\section{RÉFÉRENCES}

RC: 84007

Disponível em: https://www.nucleodoconhecimento.com.br/education-physics- 
ANDRADE, L C; ANDRADE, J.S.D; ALMEIDA S.M. Pedagogia histórico-crítica e Educação Física: o ensino das práticas corporais de aventura nos anos iniciais. Motrivivência, v. 32, n. 63, p. 01-15, 2020.

SANTOS, L. S; PORTO, C. D.; OLIVEIRA, K. E. J. Whatsapp e ciência-a conectividade científica por meio da divulgação. C\&D-Revista Eletrônica da FAINOR, v. 11, n. 2, p. 271-289, 2018.

SILVA, M. B. Contribuições Da Educação Física Escolar Para A Formação Omnilateral Do Ser Social: Uma Reflexão À Luz Da Psicologia Histórico-Cultural E Da Pedagogia Histórico-Crítica. Germinal: Marxismo e Educação em Debate, v. 9, n. 2, p. 249, 2017.

VIANA, V. N. et al. Quadras de esportes em condições de uso adequado no Brasil: influência no ensino-aprendizagem nas aulas de Educação Física (2015). Research, Society and Development, v. 9, p. e239985704-14, 2020.

VIANA, V. N.; DIAS, C. A. G. M. Educação Física e EPT. São Paulo SP: Centro de Pesquisa, 2020. 45p. Disponível em: < https://www.nucleodoconhecimento.com.br/livros/educacao/educacao-fisica-e-ept >.

DOI: 10.32749///educacao/educacao-fisica-e-ept ISBN: 978-65-86069-90-7

Soumis : Avril 2021.

Approuvé : Avril 2021.

RC: 84007

Disponível em: https://www.nucleodoconhecimento.com.br/education-physics- 